\title{
Prevalence and correlates of physical activity across kidney disease stages: an observational multicenter study
}

Thomas J. Wilkinson, $\mathrm{PhD}^{1,2}$; Amy L. Clarke, $\mathrm{PhD}^{1}$; Daniel G.D. Nixon, MSc. ${ }^{1,2}$; Katherine L. Hull, MBChB ${ }^{1,3,5}$; Yan Song, $\mathrm{PhD}^{1,4}$; James O. Burton, $\mathrm{MBChB}^{2,5}$; Thomas Yates, $\mathrm{PhD}^{2}$, 6; Alice C. Smith, $\mathrm{PhD}^{1,2}$

${ }^{1}$ Leicester Kidney Lifestyle Team, Department of Health Sciences, University of Leicester, Leicester, United Kingdom, LE1 7RH; ${ }^{2}$ NIHR Leicester Biomedical Research Centre, Leicester, United Kingdom LE5 4PW; ${ }^{3}$ University Hospitals of Leicester NHS Trust, Infirmary Square, United Kingdom, LE1 5WW; ${ }^{4}$ Nantong University Medical School, Nantong University, Nantong, China; ${ }^{5}$ Department of Cardiovascular Sciences, University of Leicester, Leicester, United Kingdom, LE1 7RH; ${ }^{6}$ Diabetes Research Centre, Leicester General Hospital, University of Leicester, Leicester, United Kingdom, LE5 4PW

\section{Corresponding author}

Dr Thomas James Wilkinson,

Leicester Kidney Lifestyle Team, Department of Health Sciences, University of Leicester and University Hospitals of Leicester, Leicester, LE1 7RH. Phone: +44 (0)116 258 4346; Email: t.j.wilkinson@le.ac.uk; ORCID: 0000-0002-7855-7752

\section{Running head}

Physical activity correlates in CKD 


\section{Abstract}

Background People with chronic kidney disease (CKD) report high levels of physical inactivity, a major modifiable risk factor for morbidity and mortality. Understanding the biological, psychosocial, and demographic causes of physical activity behaviour is essential for the development and improvement of potential health interventions and promotional initiatives. This study investigated the prevalence of physical inactivity and determined individual correlates of this behaviour in a large sample of patients across the spectrum of kidney disease.

Methods A total of 5,656 people across all stages of CKD (1-2, 3, 4-5, haemodialysis, peritoneal dialysis, and renal transplant recipients) were recruited from 17 sites in England from July 2012 to October 2018. Physical activity was evaluated using the GP Activity Questionnaire. Self-reported cardiorespiratory fitness, self-efficacy, and stage of change were also assessed. Binominal generalized linear mutually adjusted models were conducted to explore the associations between physical activity and correlate variables.

Results The prevalence of physical activity (6-34\%) was low and worsened with disease progression. Being older, female, and having a greater number of co-morbidities were associated with a greater odds of being physically inactive. Higher haemoglobin, cardiorespiratory fitness, and self-efficacy levels were associated with an increased odds of being active. Neither ethnicity nor smoking history had any effect on physical activity.

Conclusions Levels of physically inactivity are high across all stages of CKD. The identification of stage specific correlates of physical activity may help prioritize factors in 
target groups of kidney patients and improve the development and improvement of public health interventions.

Trial registration This cross-sectional observational multicenter study was registered retrospectively as ISRCTN87066351 (October 2015).

Key words Physical activity; observational; kidney disease; self-efficacy 


\section{Introduction}

Chronic kidney disease (CKD) is a serious public health issue affecting $\sim 6-8 \%$ of the UK population, with global estimates between $\sim 8-16 \%$ (1). Along with poor physical functioning and quality of life (QoL), CKD is associated with cardiovascular and metabolic morbidity and mortality risk placing a considerable strain on national health and social care resources $(1,2)$.

Physical inactivity, a physical activity level insufficient to meet present recommendations, is the 4th leading cause of death worldwide (3) and costs the UK $£ 7.4-8.3$ billion a year, including $£ 1.1$ billion to the National Health Service alone $(4,5)$. The association between physical inactivity and poor outcomes is well-established for people with $\mathrm{CKD}$, those on dialysis, and in kidney transplant recipients (RTRs) (6-9). People with kidney disease typically engage in a lower level of physical activity than the general population $(7,9-11)$ resulting in reduced neuromuscular, cardiorespiratory, and physical functioning (7), and quality of life (6). Increasing physical activity levels may confer diverse physical and psychological health benefits including moderation of long-term risks of glomerulosclerosis and progressive kidney dysfunction (12).

With the vast majority of physical activity research in kidney populations undertaken in the United States (US) and Western Europe, there remains a lack of research exploring physical activity prevalence in a UK population. The only previous UK study, conducted by Hayhurst and Ahmed (2), showed no difference in physical activity levels across disease stages (CKD, haemodialysis (HD), peritoneal dialysis (PD), and transplant patients). Whilst seemingly conflicting the wealth of previous research, this finding is likely explained by the use of a "selfcreated" un-validated composite physical activity score and a sample of just 100 participants. 
Understanding the causes of physical activity behaviour is essential for the development and improvement of potential health interventions and promotional initiatives (13). Correlates of physical activity have been studied across populations with comprehensive reviews highlighting the role of biological, psychosocial, demographic, and interpersonal factors (1317). However, limited evidence is avaliable for kidney disease, particularly in the UK.

To improve understanding of physical activity behaviour in kidney patients, the objectives of this study were to: 1) identify the prevalence of physical inactivity and 2) determine individual correlates of this behaviour in a large sample of patients across the spectrum of kidney disease. 


\section{Materials and Methods}

This cross-sectional observational multicenter study was registered retrospectively as ISRCTN87066351 (October 2015). Data were gathered between July 2012 and October 2018 from 17 sites across England, UK (Supplementary Material 1). Ethical approval was granted by the East Midlands-Leicester South Research Ethics Committee and Health Research Authority (reference: 12/EM/0184). All participants provided written informed consent. This study was performed in compliance with the Declaration of Helsinki.

\section{Participants}

Adults (aged $\geq 18$ years) with CKD able to give informed consent were eligible for inclusion. Individuals were stratified into the following cohorts: CKD Stage 1-2; CKD Stage 3; CKD Stage 4-5; prevalent (>3months) HD; PD; and RTRs according to the 'Kidney Disease: Improving Global Outcomes' definitions (18). Those recently transplanted (i.e. $<12$ weeks) were excluded as post-operative factors are likely to affect physical activity. Patients were recruited from the waiting areas of hospital outpatient clinics or dialysis treatment units. Patients were also identified from local General Practitioner (GP) practices.

\section{Outcome measures}

All outcome measures were contained in a single survey pack. Patients completed the survey pack either in the waiting areas of outpatient clinics or dialysis treatment units. Those identified by GP practices were sent the survey in the post to be completed at home.

General Practice Physical Activity Questionnaire (GPPAQ) 
Physical activity was assessed using the GPPAQ, a short questionnaire developed by the Department of Health and recommended by 'The National Institute for Health and Care Excellence' and the 'Kidney Quality Improvement Partnership'. The GPPAQ categorises individual's current physical activity as: 'active'; 'moderately active'; 'moderately inactive'; or 'inactive' $(19,20)$. Anyone scoring less than 'active' was assumed not to be meeting physical activity guidelines (20) i.e. 'insufficiently active'. Types of activities undertaken and selfreported walking pace are also recorded.

Retired and non-working respondents who do not do a sport or cycle cannot be classed as 'active' in the GPPAQ. With potential limitations in a kidney population, questionnaires were re-coded, as per Ahmad et al. (20), to include walking in the scoring, i.e. participants who reported walking at a brisk or fast pace for $\geq 3 \mathrm{~h} /$ week were re-coded as 'active'.

\section{Duke Activity Status Index (DASI)}

Physical function was measured by the DASI, a 12-item questionnaire used to assess activities of daily living (21). Total scores range from 0-58.2 with higher scores denoting greater physical capability. For better interpretation, scores were transformed into estimated peak oxygen uptake ( $\mathrm{VO}_{2}$ peak) values - a marker of cardiorespiratory fitness - as validated in CKD (22).

\section{Stages of Change Questionnaire (SOCQ)}

The SOCQ identifies respondents' state of readiness to adopt a more active lifestyle according to the 'Stages of Change Model' which consist of 5-items and forms part of a broader conceptual framework known as the Transtheoretical Model (23). The stages are: 1) precontemplation (no intention to engage in exercise behaviour); 2) contemplation (may have 
intentions to exercise in the future); 3) preparation (has started to perform limited irregular amounts of exercise); 4) action (meeting activity guidelines); and 5) maintenance (meeting guidelines for $>6$ months).

\section{Exercise Self-Efficacy Questionnaire (SEQ)}

The SEQ was used to assess an individual's confidence to regulate their exercise behaviour in the face of potential barriers representing constructs of negative affect, resisting relapse, and making time for exercise (24). Self-efficacy was rated on a 5-point Likert scale with ' 1 ' indicating 'not confident at all' to ' 5 ' indicating 'very confident'. The mean score was used as a measure of self-efficacy. The SEQ is used in other chronic disease populations with good reliability and internal consistency (24).

Clinical, co-morbidity, and demographic data

Clinical (haemoglobin $(\mathrm{Hb})$ and estimated glomerular filtration rate (eGFR)) and demographic (sex, age, smoking status, and ethnicity) data were taken from recent medical records and selfreported responses. Kidney function was determined by eGFR using the 'Modification of Diet in Renal Disease Formula'. Co-morbidities were recorded based on a composite of patient selfreport and medical notes. For RTRs, months since transplant and donor type were recorded.

\section{Statistical analysis}

Descriptive and frequency statistics were used to describe patient characteristics. Dichotomous and categorical variables are presented as percentages and continuous variables presented as median (interquartile range [IQR]) for non-normally distributed data. Participants without completed survey packs were excluded from the analysis and missing data for other variables 
were analysed listwise. No data imputations were performed. Missing data frequency can be found in Supplementary Material 2. All statistical analyses were performed using IBM SPSS (Version 24, IBM, USA). Statistical significance was accepted as a $P$ value of $<0.05$.

A multinomial generalized linear model was used to explore differences in physical activity status across disease stages with CKD Stage 1-2 used as a reference group adjusting for age and sex. Binominal generalized linear mutually adjusted models were conducted to explore the associations between physical activity (coded as a dichotomous variable) and demographics (age, sex, ethnicity, smoking status), clinical parameters $(\mathrm{Hb})$, total number of co-morbidities (in addition to kidney disease), $\mathrm{VO}_{2}$ peak, self-efficacy, and stages of change. Stages of change were coded as non-receptive (pre-contemplation) or receptive (contemplation, preparation, action and maintenance). Age, $\mathrm{Hb}$, the total number of co-morbidities, and $\mathrm{VO}_{2}$ peak were defined as continuous variables. Self-efficacy scores were treated as a continuous value as per the original citation (24). Interaction analyses were conducted to assess whether disease stage modified these associations. Significant disease stage interactions were further investigated through stratified analysis with age and $\mathrm{VO}_{2}$ peak coded as a dichotomous variables based on the median of the total sample. Unless stated, results are expressed as mutually adjusted odds ratio $(\mathrm{OR})$ and $95 \%$ confidence intervals. An OR of $>1$ indicates a greater odds of being classified as physically 'inactive'. An OR $<1$ represents a smaller odds of being classified as physically 'inactive'. For continuous variables, odds are reported per 1 unit change. 


\section{Results}

\section{Participant characteristics}

5,656 people were recruited from 17 sites in England. Data from 398 cases were excluded (no eGFR recorded, $n=380$; no data recorded, $n=13$; unreliable data, $n=5$ ) leaving a total of 5258 cases for analysis. Information on recruiting centres is included in Supplementary Material 2. Full clinical and demographic characteristics are shown in Table 1. Females represented $42 \%$ of the total sample. People with CKD Stage 1-2 were younger with the oldest participants found in CKD Stage 4-5. 23\% of the sample were from a non-White background. In RTRs, the median time post-transplant was 41.0 (IQR 105.0) months. The majority (66\%) of transplants were from cadaveric donation, followed by unrelated living (21\%) and related living donation $(13 \%)$.

\section{Physical inactivity prevalence}

The prevalence of insufficient physical activity was high and worsened with disease progression (Figure 1). Patients in CKD Stage 1-2 were most active (34\% sufficiently active). Physical activity decreased from CKD Stage 3 (17\% active) through to CKD Stage 4-5 (11\% active) before reaching a nadir in people requiring dialysis (only 6\% HD and 8\% PD patients were active). Physical activity of patients in CKD Stage 4-5, HD, and PD were significantly different $(P<0.001)$ than CKD Stage $1-2.27 \%$ of RTRs were sufficiently active. 
The frequency of physical activities is reported in Figure 2. Walking was the most popular form of activity across all stages. Full physical activity data can be found in Supplementary Material 3.

\section{Correlates of physical inactivity}

Table 2 shows the association of demographic, clinical, physical, and psychological characteristics with the likelihood of being physically inactive in the combined study cohort. Being older, female, and having a greater number of co-morbidities were associated with a greater odds of being inactive. Higher $\mathrm{Hb}$ and $\mathrm{VO}_{2}$ peak levels were associated with a reduced odds of being inactive. Patients in a receptive stage of change and with higher levels of selfefficacy had greater odds of being active. Neither ethnicity nor smoking history had any effect on physical activity.

Interaction analysis revealed that disease stage modified some of these associations, outlined in Table 2. The direction of the interactions is displayed in Figure 3 (and Supplementary Material 4). The effect of age was most pronounced in CKD Stage 4-5; those older than 61 years were 5.5 times more likely to be inactive than those $\leq 61$ years. Although females were less active than males across all disease stages, in those on $\mathrm{HD}$, females were 5.0 times more likely to be inactive than males. $\mathrm{VO}_{2}$ peak levels had a greater effect on activity level in those on dialysis and RTRs; in these groups patients with a $\mathrm{VO}_{2}$ peak $\leq 22.3 \mathrm{ml} \cdot \mathrm{kg} \cdot \mathrm{min}^{-1}$ were between 4.9 and 18.4 times more likely to be inactive than those with a $\mathrm{VO}_{2}$ peak $>22.3 \mathrm{ml} \cdot \mathrm{kg} \cdot \mathrm{min}^{-1}$. 


\section{Discussion}

With more than 5000 participants, we present the largest analysis of physical activity behaviour in a UK kidney population. The key findings are: 1) physical inactivity is highly prevalent across all stages of CKD 2) physical activity levels worsen with disease progression; 3) physical activity levels are improved in those with a kidney transplant; 4) being older, female, having a lower $\mathrm{Hb}$, lower self-efficacy, lower cardiorespiratory fitness, and being in a "nonreceptive' stage of change are associated with being inactive.

Despite the growing evidence supporting the importance of physical activity in kidney disease $(6,7)$, inactivity was highly prevalent across all stages of CKD. In support of previous literature (9), we observed a decline in physical activity with kidney disease progression, reaching a nadir in those on dialysis. In the UK general population, physical activity data using the GPPAQ is limited and data concerning adherence to recommended levels of physical activity is variable, however it is estimated that $\sim 35-43 \%$ of adults are 'inactive' $(4,19)$. In our sample, with the exception of those with CKD Stage 1-2 and RTRs (42-46\% inactive), physical inactivity was considerably higher in people with more advanced disease. In CKD Stage 4-5 and those on dialysis the prevalence of people defined as 'active' was only $6-11 \%$; this is less than a cohort of 114 mixed cancer (bowel, breast, or prostate) patients using the GPPAQ (25).

It is perhaps unsurprising that physical activity levels worsen as CKD progresses given the increasing symptom burden (26) and reductions in physical function aggravated by anaemia, metabolic acidosis, inflammation, and malnutrition (9). People with CKD Stages 1-2 generally have less disease burden and are treated in primary care by a General Practitioner without the 
need for specialist referral. In our study, this group of 'early' CKD patients were younger with a lower prevalence of additional comorbidities.

Physical activity was lowest in patients requiring dialysis with $94 \%$ of HD patients and $92 \%$ of PD patients 'insufficiently active'. Low physical activity in dialysis patients is welldescribed (8-11, 27-29) with several causes including uraemia, fatigue, comorbidity burden, anaemia, and depression $(30,31)$. In HD patients, post-dialysis fatigue as well the logistical processes of dialysing several days per week negatively impact physical activity (32). Patients on PD may feel discouraged from participating in physical activity due to concerns about the development of hernias and leaks, or uncertainty surrounding appropriate exercise regimens (28). Accordingly, such levels of inactivity are worrying given the association with mortality in HD and PD (33).

Physical activity was higher in transplant patients relative to those with advanced CKD. Such 'recovery' of physical activity following a transplant has been previously observed (34-36). Nielens et al. (37) reported an immediate decrease in physical activity one-month posttransplant, followed by increased physical activity and a plateau after one year. All RTRs in the present study were $>12$-weeks post-transplant thus negotiating any initial decline. Nonetheless, relatively few met national recommendations. Although receipt of a kidney transplant has been shown to improve quality of life and reduce the risk of ESKD-related outcomes (38), new (e.g., fear of harming graft, immunosuppression-related side effects) and existing pre-transplant (e.g., fatigue, low physical condition) barriers remain $(36,39,40)$. The low rates of physical activity in RTRs observed are of concern because, along with improved physical and psychological quality of life, greater physical activity is associated with improved graft function $(6,9,34-36)$. 
It is well-recognized in epidemiological research that increasing age is associated with reduced physical activity (13-17). We observed an inverse relationship between age and physical activity across all stages, although particularly evident in CKD Stage 4-5. Likely age-related factors include declines in health status, mobility, or motivation (16), intensified with reductions in physical and kidney function. Consistent with research in both large general (1317) and kidney population studies (29), sex emerged as a strong correlate of physical activity. In people with non-dialysis $\mathrm{CKD}$, males were approximately three times more likely to be active than females, and in those undergoing HD, males were over five times more likely to be active. Multiple explanations like family and societal roles, psychological issues, and life conditions may account for these differences (15).

Regardless of CKD stage, the physical function and cardiorespiratory fitness of our sample were poor. We observed that a higher $\mathrm{VO}_{2 \text { peak }}$ was associated with a greater likelihood of being active in patients with ESKD (PD and HD) and RTRs. Whilst this relationship is likely bidirectional, having sufficient cardiorespiratory fitness is an important criterion of the capability to be active (39) and low cardiorespiratory fitness is associated with impaired physical functioning (9). Segura-Ortiz et al. (27) found poor physical function was associated with reduced physical activity, and in HD patients (41) and RTRs (36) physical activity has been closely associated with physical function. Importantly, whilst impaired fitness may hinder patients from undertaking physical activity, being inactive contributes to further reductions in physical capability (9). Interestingly, our analysis suggests that physical fitness may not be an important factor in determining physical activity in those with non-dialysis dependent CKD. 
The concentration of oxygen-carrying $\mathrm{Hb}$ is a well-established limiting parameter of cardiorespiratory capacity. With anaemia a common complication of $\mathrm{CKD}$, it is unsurprising that low $\mathrm{Hb}$ levels have been associated with inactivity (9). We found higher $\mathrm{Hb}$ levels were associated with a greater odds of being active regardless of CKD stage. This supports previous research $(2,27,30)$ although contrasts others $(2,42)$.

From a psychological perspective, self-efficacy is purportedly the most important predictor of engaging in and long-term maintenance of physical activity $(14,43)$. Self-efficacy refers to an individual's beliefs about their capability to perform a particular behaviour (44), and in our cohort, the confidence to regulate exercise behaviour in the face of potential barriers. We found higher self-efficacy was associated with a greater odds of being active across all CKD groups. Previous studies in kidney populations is limited, however studies in RTRs $(9,35,39,40)$, those on dialysis $(45,46)$, and unspecified kidney disease (47) have shown associations between self-efficacy and physical activity. With growing evidence underlining its importance, strategies to promote self-efficacy may increase physical activity engagement $(9,35,39,47)$.

Patients in a 'receptive' stage of change were more likely to be active. The 'Stages of Change' model from Prochaska and DiClemente's Transtheoretical Model has been used extensively to study health-related behaviours including physical activity (48). The model postulates that individuals engaging in a new behaviour move through non-receptive to receptive stages (24). For those in a non-receptive stage, the will to abstain from physical activity is greater than the self-efficacy for it (48). In these people, traditional interventions are unsuitable and targeting of stage-specific interventions is required $(24,48)$. Physical activity promotion should focus on 'building readiness' to change and people may benefit most from informational, 
educational, and motivational experiences designed to increase the appeal and expectations of physical activity $(24,48)$.

Despite its large and diverse sample, our study is limited by its cross-sectional design, a common limitation in physical activity literature (17) precluding determination of causality. Nonetheless, this design can provide evidence about potential mediators for the planning of interventions and may help prioritise factors in target groups of kidney patients. Such design also allows several variables to be assessed at low cost providing evidence for intervention design improvement (49). The use of self-report is widely used for physical activity assessment, despite various shortcomings (e.g., recall bias, misinterpretation) (8). In the current study, the GPPAQ was used to measure physical activity. Whilst easy to administer, research has revealed a sensitivity of between $19-46 \%$ and specificity of $50-85 \%$ (classed as 'active') compared to accelerometery $(20,50)$. It is important to note that our population consisted of patients willing to engage in the research study and return a completed survey. Such sampling bias may be evident in the somewhat lower prevalence of comorbidities such as hypertension, diabetes, and CVD; this may reduce the generalizability of our findings to a wider patient population.

The solution to combating physical inactivity is complex, multifaceted, and likely dependent on each individual. Our results show strategies promoting self-efficacy may be key to increasing physical activity. Self-efficacy arises from four primary sources: mastery experiences, social modelling, social persuasion, and the interpretation of physiological and emotional responses $(43,44)$. Whilst beyond the scope of this work, each of these can targeted to maximize physical activity engagement. For example, facilitated by a healthcare professional, the setting of challenging, but reachable, physical activity goals can be an effective method of ensuring regular successful experience. Social or group activities may aid 
in social modelling and persuasion, and being able to interpret aches and pains associated with activity after a considerable period of time as positive responses enhances self-efficacy (43). With only small increases in activity beneficial, nephrologists and healthcare professionals should engage and discuss the risks and benefits of physical activity with their patients, identify barriers, and highlight its relative safety and the adverse outcomes associated with inactivity. Although proper infrastructure and specialist supervision to facilitate exercise programs for CKD patients are largely lacking, a simple prescription of short-term home-based aerobic exercise at moderate intensity, such as brisk walking or cycling for 30 to 60 minutes at least three times per week may form the basis of a cheap and safe programme. Structured exercise incorporating strength and balance training may be introduced in appropriate patients (9).

In conclusion, in the largest UK cohort of its kind, we established that physical inactivity is highly prevalent across all stages of kidney disease, reaching a nadir in those requiring dialysis. We were able to identify stage specific correlates of physical activity. Our findings are important as physical inactivity is a major modifiable risk factor for morbidity and mortality, and a better understanding of the causes of physical activity behaviour is essential for the development and improvement of public health interventions. 


\section{Declarations}

\section{Acknowledgments}

This report is independent research supported by the National Institute for Health Research Leicester Biomedical Research Centre. The views expressed are those of the author(s) and not necessarily those of the NHS, the National Institute for Health Research Leicester BRC or the Department of Health. We would like to acknowledge the numerous research facilitators at each site who contributed to the collection of data in this study.

\section{Competing interests}

The authors declare that they have no competing interests. T.Y. reports grants from NIHR during the conduct of the study

\section{Author contributions}

A.C.S. and J.O.B. contributed to the research idea and study design. T.J.W., A.L.C., D.G.D.N., K.L.H., and Y.S. contributed to the generation/collection of data. T.J.W., A.L.C., D.G.D.N., K.L.H., Y.S. J.O.B., T.Y., and A.C.S. helped in data analysis/interpretation. T.J.W. and T.Y. performed the statistical analysis. A.C.S., T.Y., and J.O.B. were involved in supervision or mentorship. T.J.W. drafted the manuscript. Each author contributed important intellectual content during manuscript revision and accepts responsibility for the overall work by ensuring that questions pertaining to the accuracy or integrity of any portion of the work are appropriately investigated and resolved.

\section{Funding}


This research was part-funded by the Stoneygate Trust and a British Renal Society Research Grant (2011). A.L.C. was partly supported by a Kidney Research UK Innovation Grant (IN7/2014), J.O.B. was supported by a NIHR Clinician Scientist Award (CS-2013-13-014) and K.L.H. received a Kidney Research UK Medical Student Bursary for 2012-2013.

\section{Availability of data and materials}

The datasets used and/or analysed during the current study are available from the corresponding author on reasonable request. 


\section{References}

1. Jha V, Garcia-Garcia G, Iseki K, et al. Chronic kidney disease: global dimension and perspectives. Lancet 2013; 382: 260-272

2. Hayhurst WS, Ahmed A. Assessment of physical activity in patients with chronic kidney disease and renal replacement therapy. SpringerPlus 2015; 5: 961

3. World Health Organisation. Global status report on noncommunicable diseases 2014. Available at https://www.who.int/nmh/publications/ncd-status-report-2014/en/

4. Public Health England. Health matters: getting every adult active every day 2016. Available at https://www.gov.uk/government/publications/health-matters-getting-every-adultactive-every-day

5. Foster J, Thompson K, Harkin J. Let's Get Moving: Commissioning Guidance: a New Physical Activity Care Pathway for the NHS: Department of Health; 2009. Available at https://assets.publishing.service.gov.uk/government/uploads/system/uploads/attachment_data /file/216262/dh_133101.pdf

6. MacKinnon HJ, Wilkinson TJ, Clarke AL, et al. The association of physical function and physical activity with all-cause mortality and adverse clinical outcomes in nondialysis chronic kidney disease: a systematic review. Ther Adv Chronic Dis 2018; 9: 209-226

7. Beddhu S, Baird BC, Zitterkoph J, Neilson J, Greene T. Physical Activity and Mortality in Chronic Kidney Disease (NHANES III). Clin J Am Soc Nephrol 2009; 4: 1901-1906

8. Painter P, Roshanravan B. The association of physical activity and physical function with clinical outcomes in adults with chronic kidney disease. Curr Opin Nephrol Hypertens $2013 ; 22: 615-23$

9. Zelle DM, Klaassen G, van Adrichem E, Bakker SJL, Corpeleijn E, Navis G. Physical inactivity: a risk factor and target for intervention in renal care. Nat Rev Nephrol 2017; 13 : $152-168$ 
10. Johansen KL, Chertow GM, Ng AV, et al. Physical activity levels in patients on hemodialysis and healthy sedentary controls. Kidney Int 2000; 57: 2564-2570

11. Painter P, Ward K, Nelson RD. Self-reported physical activity in patients with end stage renal disease. Nephrol Nurs J 2011; 38: 139-147

12. Robinson-Cohen C, Katz R, Mozaffarian D, et al. Physical Activity and Rapid Decline in Kidney Function Among Older Adults. Arch Intern Med 2009; 169: 2116-2123

13. Bauman AE, Reis RS, Sallis JF, et al. Correlates of physical activity: why are some people physically active and others not? Lancet 2012; 380: 258-271

14. Trost SG, Owen N, Bauman AE, Sallis JF, Brown W. Correlates of adults' participation in physical activity: review and update. Med Sci Sports Exerc 2002; 34: 1996-2001

15. Murtagh EM, Murphy MH, Murphy NM, Woods C, Nevill AM, Lane A. Prevalence and correlates of physical inactivity in community-dwelling older adults in Ireland. PloS One 2015; 10: e0118293

16. Souza AM, Fillenbaum GG, Blay SL. Prevalence and correlates of physical inactivity among older adults in Rio Grande do Sul, Brazil. PloS One 2015; 10: e0117060

17. Bauman AE, Sallis JF, Dzewaltowski DA, Owen N. Toward a better understanding of the influences on physical activity: the role of determinants, correlates, causal variables, mediators, moderators, and confounders. Am J Prev Med 2002; 23: 5-14

18. Levey AS, Eckardt KU, Tsukamoto Y, et al. Definition and classification of chronic kidney disease: a position statement from Kidney Disease: Improving Global Outcomes (KDIGO). Kidney Int 2005; 67: 2089-2100

19. Heron N, Tully MA, McKinley MC, Cupples ME. Physical activity assessment in practice: a mixed methods study of GPPAQ use in primary care. BMC Fam Pract 2014; 15:11 
20. Ahmad S, Harris T, Limb E, et al. Evaluation of reliability and validity of the General Practice Physical Activity Questionnaire (GPPAQ) in 60-74 year old primary care patients. BMC Fam Pract 2015; 16: 113

21. Hlatky MA, Boineau RE, Higginbotham MB, et al. A brief self-administered questionnaire to determine functional capacity (the Duke Activity Status Index). Am J Cardiol 1989; 64: 651-654

22. Ravani P, Kilb B, Bedi H, Groeneveld S, Yilmaz S, Mustata S, Alberta Kidney Disease Network. The Duke Activity Status Index in patients with chronic kidney disease: a reliability study. Clin J Am Soc Nephrol 2012; 7: 573-580

23. Prochaska JO, DiClemente CC. Transtheoretical therapy: Toward a more integrative model of change. Psychother Theor Res Pract Train 1982; 19: 276-288

24. Marcus BH, Selby VC, Niaura RS, Rossi JS. Self-efficacy and the stages of exercise behavior change. Res Q Exerc Sport 1992; 63: 60-66

25. Yang DD, Hausien O, Aqeel M, et al. Physical activity levels and barriers to exercise referral among patients with cancer. Patient Educ Couns 2017; 100: 1402-1407

26. Brown SA, Tyrer FC, Clarke AL, et al. Symptom burden in patients with chronic kidney disease not requiring renal replacement therapy. Clin Kidney J 2017; 10: 788-796

27. Segura-Ortí E, Gordon P, Doyle J, Johansen K. Correlates of Physical Functioning and Performance Across the Spectrum of Kidney Function. Clin Nurs Res 2018; 27: 579-596

28. Thangarasa T, Imtiaz R, Hiremath S, Zimmerman D. Physical activity in patients treated with peritoneal dialysis: a systematic review and meta-analysis. Can J Kidney Health Dis 2018; 5: 2054358118779821

29. Johansen KL, Chertow GM, Kutner NG, Dalrymple LS, Grimes BA, Kaysen GA. Low level of self-reported physical activity in ambulatory patients new to dialysis. Kidney Int 2010; 78: $1164-1170$ 
30. Zamojska S, Szklarek M, Niewodniczy M, Nowicki M. Correlates of habitual physical activity in chronic haemodialysis patients. Nephrol Dial Transplant 2006; 21: 1323-1327

31. Anand S, Johansen KL, Grimes B, et al. Physical activity and self-reported symptoms of insomnia, restless legs syndrome, and depression: The comprehensive dialysis study. Hemodial Int 2013; 17: 50-58

32. Majchrzak KM, Pupim LB, Chen K, et al. Physical activity patterns in chronic hemodialysis patients: comparison of dialysis and nondialysis days. J Ren Nutr 2005; 15: 217224

33. Johansen KL, Kaysen GA, Dalrymple LS, et al. Association of physical activity with survival among ambulatory patients on dialysis: the comprehensive dialysis study. Clin J Am Soc Nephrol 2013; 8: 248-253

34. Rosas SE, Reese PP, Huan Y, Doria C, Cochetti PT, Doyle A. Pretransplant Physical Activity Predicts All-Cause Mortality in Kidney Transplant Recipients. Am J Nephrol 2012; $35: 17-23$

35. Gordon EJ, Prohaska TR, Gallant MP, et al. Prevalence and determinants of physical activity and fluid intake in kidney transplant recipients. Clin Transplant 2010; 24: E69-81

36. Takahashi A, Hu SL, Bostom A. Physical activity in kidney transplant recipients: a review. Am J Kidney Dis 2018; 72: 433-443

37. Nielens H, Lejeune TM, Lalaoui A, Squifflet JP, Pirson Y, Goffin E. Increase of physical activity level after successful renal transplantation: a 5 year follow-up study. Nephrol Dial Transplant 2001; 16:134-140

38. Stoumpos S, Jardine AG, Mark PB. Cardiovascular morbidity and mortality after kidney transplantation. Transplant Int 2015; 28:10-21 
39. van Adrichem EJ, van de Zande SC, Dekker R, Verschuuren EA, Dijkstra PU, van der Schans CP. Perceived barriers to and facilitators of physical activity in recipients of solid organ transplantation, a qualitative study. PloS One 2016; 11: e0162725

40. Zelle DM, Corpeleijn E, Klaassen G, Schutte E, Navis G, Bakker SJ. Fear of movement and low self-efficacy are important barriers in physical activity after renal transplantation. PloS One 2016; 11: e0147609

41. O'Hare AM, Bacchetti P, Segal M, Hsu C-y, Johansen KL. Factors associated with future amputation among patients undergoing hemodialysis: results from the Dialysis Morbidity and Mortality Study Waves 3 and 4. Am J Kidney Dis 2003; 41: 162-170

42. Araújo Filho JCd, Amorim CTd, Brito ACNdL, Oliveira DSd, Lemos A, Marinho PÉdM: Physical activity level of patients on hemodialysis: a cross-sectional study. Fisioter. Pesqui 2016; 23: 234-240

43. McAuley, Szabo, Gothe, Olson EA. Self-efficacy: Implications for Physical Activity, Function, and Functional Limitations in Older Adults. Am J Lifestyle Med 2011; 5: $10.1177 / 1559827610392704$

44. Bandura A: Self-efficacy: toward a unifying theory of behavioral change. Psychol Rev 1977 84: 191-215

45. Patterson MS, Umstattd Meyer MR, Beaujean AA, Bowden RG. Using the social cognitive theory to understand physical activity among dialysis patients. Rehabil Psychol 2014; 59: $278-288$

46. Delgado C, Johansen KL. Barriers to exercise participation among dialysis patients. Nephrol Dial Transplant 2011; 27:1152-1157

47. Daniali SS, Darani FM, Eslami AA, Mazaheri M. Relationship between Self-efficacy and Physical Activity, Medication Adherence in Chronic Disease Patients. Adv Biomed Res 2017; 29: 63 
48. Ronda G, Van Assema P, Brug J. Stages of change, psychological factors and awareness of physical activity levels in the Netherlands. Health Promot Int 2001; 16: 305-314 49. Sallis JF, Saelens BE. Assessment of physical activity by self-report: status, limitations, and future directions. Res Q Exerc Sport 2000; 71: 1-14

50. Fitzgerald L, Ozemek C, Jarrett H, Kaminsky LA. Accelerometer validation of questionnaires used in clinical settings to assess MVPA. Med Sci Sports Exerc 2015; 47: 15381542 
Tables

Table 1. Patient demographic and clinical characteristics

\begin{tabular}{|c|c|c|c|c|c|c|}
\hline \multirow{2}{*}{$\mathrm{N}=5258$} & CKD Stage 1-2 & CKD Stage 3 & CKD Stage 4-5 & HD & PD & RTRs \\
\hline & $n=281$ & $n=752$ & $n=646$ & $n=1,155$ & $n=184$ & $n=2,240$ \\
\hline \multicolumn{7}{|l|}{ Sex } \\
\hline Male (n, \%) & $137(49 \%)$ & $363(49 \%)$ & $376(58 \%)$ & $726(64 \%)$ & $122(67 \%)$ & $1289(59 \%)$ \\
\hline Female $(\mathrm{n}, \%)$ & $144(51 \%)$ & $383(51 \%)$ & $270(42 \%)$ & $405(36 \%)$ & $61(33 \%)$ & $909(41 \%)$ \\
\hline \multicolumn{7}{|l|}{ Age (years) } \\
\hline Median (IQR) & $47.0(26.0)$ & $72.0(18.0)$ & $73.0(21)$ & $65.0(21.0)$ & $64.0(22.0)$ & $53.0(20.0)$ \\
\hline$\leq 61$ years $(\mathrm{n}, \%)^{\mathrm{a}}$ & $212(76 \%)$ & $179(24 \%)$ & $176(27 \%)$ & $469(42 \%)$ & $78(43 \%)$ & $1532(71 \%)$ \\
\hline$>61$ years $(n, \%)$ & $68(24 \%)$ & $559(76 \%)$ & $468(73 \%)$ & $656(58 \%)$ & $104(57 \%)$ & $636(29 \%)$ \\
\hline \multicolumn{7}{|l|}{ Ethnicity } \\
\hline White British (n, \%) & $208(83 \%)$ & $413(89 \%)$ & $508(79 \%)$ & $419(49 \%)$ & $103(66 \%)$ & $1503(74 \%)$ \\
\hline White other (n, \%) & $7(3 \%)$ & $18(4 \%)$ & $14(2 \%)$ & $27(3 \%)$ & $6(4 \%)$ & $143(7 \%)$ \\
\hline Indian $(\mathrm{n}, \%)$ & $17(7 \%)$ & $19(2 \%)$ & $37(6 \%)$ & $114(13 \%)$ & $14(9 \%)$ & $39(2 \%)$ \\
\hline Pakistani (n, \%) & $0(0 \%)$ & $2(0 \%)$ & $3(1 \%)$ & $25(3 \%)$ & $6(4 \%)$ & $19(1 \%)$ \\
\hline Asian other $(\mathrm{n}, \%)$ & $4(2 \%)$ & $8(2 \%)$ & $7(1 \%)$ & $39(5 \%)$ & $8(5 \%)$ & $136(7 \%)$ \\
\hline Caribbean $(n, \%)$ & $5(2 \%)$ & $3(1 \%)$ & $2(0 \%)$ & $90(11 \%)$ & $9(6 \%)$ & $69(3 \%)$ \\
\hline African $(\mathrm{n}, \%)$ & $2(1 \%)$ & $0(0 \%)$ & $7(1 \%)$ & $96(11 \%)$ & $7(4 \%)$ & $30(1 \%)$ \\
\hline Other $(\mathrm{n}, \%)$ & $7(3 \%)$ & $2(0 \%)$ & $5(1 \%)$ & $42(5 \%)$ & $3(2 \%)$ & $93(5 \%)$ \\
\hline \multicolumn{7}{|l|}{ Smoking status } \\
\hline Current smoker (n, \%) & $47(17 \%)$ & $65(9 \%)$ & $68(11 \%)$ & $107(10 \%)$ & $13(7 \%)$ & $161(7 \%)$ \\
\hline Never smoked (n, \%) & $122(45 \%)$ & $324(45 \%)$ & $256(41 \%)$ & $556(50 \%)$ & $88(49 \%)$ & $1262(58 \%)$ \\
\hline Previous smoker $(\mathrm{n}, \%)$ & $105(38 \%)$ & $334(46 \%)$ & $295(48 \%)$ & $453(41 \%)$ & $80(44 \%)$ & $736(34 \%)$ \\
\hline \multicolumn{7}{|l|}{$\operatorname{eGFR}\left(\mathrm{ml} / \mathrm{min} / 1.73 \mathrm{~m}^{2}\right)$} \\
\hline Median (IQR) & $78.0(23.0)$ & $44.0(15.0)$ & $20.2(10.0)$ & $6.5(4.0)$ & $7.0(5.0)$ & $46.0(26)$ \\
\hline \multicolumn{7}{|l|}{$H b(g / d L)$} \\
\hline Median (IQR) & $13.4(2.4)$ & $12.7(2.3)$ & $11.6(2.1)$ & $11.2(1.8)$ & $10.9(2.2)$ & $12.7(2.5)$ \\
\hline Diabetes (n, \%) & $33(12 \%)$ & $187(26 \%)$ & $217(35 \%)$ & $341(39 \%)$ & $50(34 \%)$ & $374(22 \%)$ \\
\hline $\operatorname{CVD}(\mathbf{n}, \%)$ & $20(7 \%)$ & $182(25 \%)$ & $146(24 \%)$ & $201(23 \%)$ & $25(17 \%)$ & $175(10 \%)$ \\
\hline Hypertension (n, \%) & $119(47 \%)$ & $292(71 \%)$ & $481(79 \%)$ & $563(64 \%)$ & $91(62 \%)$ & $1275(73 \%)$ \\
\hline Total no. comorbidities ${ }^{b}$ & & & & & & \\
\hline
\end{tabular}




\begin{tabular}{|c|c|c|c|c|c|c|}
\hline Mean (SD) & $1.1(1.1)$ & $1.8(1.3)$ & $1.9(1.3)$ & $1.0(1.0)$ & $1.2(1.1)$ & $1.4(1.1)$ \\
\hline Median (IQR) & $1.0(2.0)$ & $2.0(2.0)$ & $2.0(2.0)$ & $1.0(2.0)$ & $1.0(2.0)$ & $1.0(1.0)$ \\
\hline \multicolumn{7}{|l|}{ DASI } \\
\hline Median (IQR) & $31.5(32.4)$ & $31.7(31.8)$ & $26.0(31.3)$ & $18.0(20.5)$ & $23.2(24.0)$ & $38.2(32.4)$ \\
\hline \multicolumn{7}{|l|}{ VO2peak (ml.kg. $\left.\mathrm{min}^{-1}\right)$} \\
\hline Median (IQR) & $23.1(13.9)$ & $23.2(13.7)$ & $20.8(13.4)$ & $17.3(8.8)$ & $19.6(10.3)$ & $26.0(13.9)$ \\
\hline$\leq 22.3$ ml.kg. $\min ^{-1}(\mathrm{n}, \%)^{\mathrm{c}}$ & $123(44 \%)$ & $351(47 \%)$ & $363(56 \%)$ & $818(74 \%)$ & $120(66 \%)$ & $750(37 \%)$ \\
\hline$>22.3$ ml.kg. $\min ^{-1}(\mathrm{n}, \%)$ & $158(56 \%)$ & $400(53 \%)$ & $283(44 \%)$ & $289(26 \%)$ & $63(34 \%)$ & $1278(63 \%)$ \\
\hline \multicolumn{7}{|l|}{ Stage of Change } \\
\hline Pre-contemplation (n, \%) & $42(15 \%)$ & $261(37 \%)$ & $291(47 \%)$ & $460(44 \%)$ & $67(38 \%)$ & $335(18 \%)$ \\
\hline Contemplation (n, \%) & $63(23 \%)$ & $111(16 \%)$ & $108(18 \%)$ & $215(20 \%)$ & $44(25 \%)$ & $436(23 \%)$ \\
\hline Preparation (n, \%) & $58(21 \%)$ & $118(17 \%)$ & $105(17 \%)$ & $202(19 \%)$ & $34(19 \%)$ & $465(25 \%)$ \\
\hline Action (n, \%) & $21(8 \%)$ & $30(4 \%)$ & $19(3 \%)$ & $47(4 \%)$ & $7(4 \%)$ & $157(8 \%)$ \\
\hline Maintenance (n, \%) & $89(33 \%)$ & $195(27 \%)$ & $94(15 \%)$ & $129(12 \%)$ & $23(13 \%)$ & $471(25 \%)$ \\
\hline \multicolumn{7}{|l|}{ Exercise SEQ } \\
\hline Median (IQR) / 5 & $3.0(1.5)$ & $2.2(2.2)$ & $2.2(2.0)$ & $1.2(1.6)$ & $1.6(1.7)$ & $2.4(2.0)$ \\
\hline
\end{tabular}

Data shown as frequencies or median (with interquartile range $(\mathrm{IQR})$ ), unless otherwise stated. $\mathrm{CKD}=$ chronic kidney disease; $\mathrm{HD}=$ haemodialysis; $\mathrm{PD}=$ peritoneal dialysis; RTRs = renal transplant recipients; $\mathrm{SD}=$ standard deviation; $\mathrm{IQR}=$ interquartile range; eGFR = estimated glomerular filtration rate; $\mathrm{Hb}=$ haemoglobin; $\mathrm{CVD}=$ previous heart disease; DASI = Duke Activity Status Index; $\mathrm{VO}_{2}$ peak $=$ calculated from DASI; SEQ $=$ Self-Efficacy Questionnaire. ${ }^{\mathrm{a}}=61$ years denotes median of total sample; ${ }^{\mathrm{b}}=$ Total no. of additional comorbidities (excluding kidney disease); $\mathrm{c}=22.3 \mathrm{ml} . \mathrm{kg}$. $\mathrm{min}^{-1}$ denotes median of total sample. Percentage of missing excluded from percentage accumulation of other variables 
Table 2. Correlates of physical inactivity and interactions with disease group

\begin{tabular}{|c|c|c|c|}
\hline $\mathrm{N}=2,956$ & OR $(95 \% \mathrm{CI})$ & $\begin{array}{l}\text { Main effect } \\
P \text { value }\end{array}$ & $\begin{array}{c}\text { Interaction } \\
P \text { value }\end{array}$ \\
\hline Age & 1.029 (1.021 to 1.037$)$ & $<0.001 *$ & $0.001 *$ \\
\hline $\begin{array}{l}\text { Sex [Male (reference)] } \\
\text { Female }\end{array}$ & $\begin{array}{c}1 \\
1.668(1.340 \text { to } 2.076)\end{array}$ & $<0.001 *$ & $0.03 *$ \\
\hline $\begin{array}{l}\text { Ethnicity [White British (reference)] } \\
\text { Non-white }\end{array}$ & $\begin{array}{c}1 \\
1.270(.930 \text { to } 1.733)\end{array}$ & 0.13 & 0.71 \\
\hline Hb & $.932(.879$ to .989$)$ & $0.02 *$ & 0.24 \\
\hline $\begin{array}{l}\text { Smoking status [Never smoked (reference)] } \\
\text { Current smoker } \\
\text { Previous smoker }\end{array}$ & $\begin{array}{c}1 \\
1.058(.738 \text { to } 1.517) \\
.974(.779 \text { to } 1.219)\end{array}$ & 0.91 & 0.85 \\
\hline Total co-morbidities ${ }^{\text {a }}$ & $1.172(1.057$ to 1.300$)$ & $0.003 *$ & 0.37 \\
\hline $\begin{array}{l}\text { Stage of change [Non-receptive (reference)] } \\
\text { Receptive }\end{array}$ & $\begin{array}{c}1 \\
.352(.247 \text { to } .501)\end{array}$ & $<0.001 *$ & 0.29 \\
\hline SEQ & $.703(.642$ to .770$)$ & $<0.001 *$ & 0.34 \\
\hline $\mathrm{VO}_{2}$ peak & $.923(.909$ to .937$)$ & $<0.001 *$ & $<0.001 *$ \\
\hline
\end{tabular}

Data displayed as mutually adjusted Odds Ratio (OR) with $95 \%$ confidence interval (95\% CI) for main effects; an OR of $>1$ indicates greater odds of being classified as physically 'inactive'. Significant interaction denotes modifying effect of chronic kidney disease (CKD) group. The direction of the significant interactions for age, sex, and $\mathrm{VO}_{2}$ peak are displayed in Figure 3. Significance denoted by * and set at $P<0.05$.

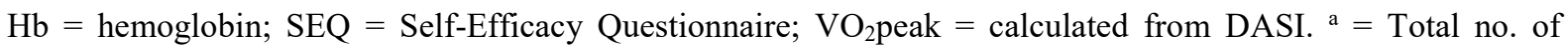
additional comorbidities (excluding kidney disease) 


\section{Figures and legends}

Figure 1. Levels of physical activity status across disease stages

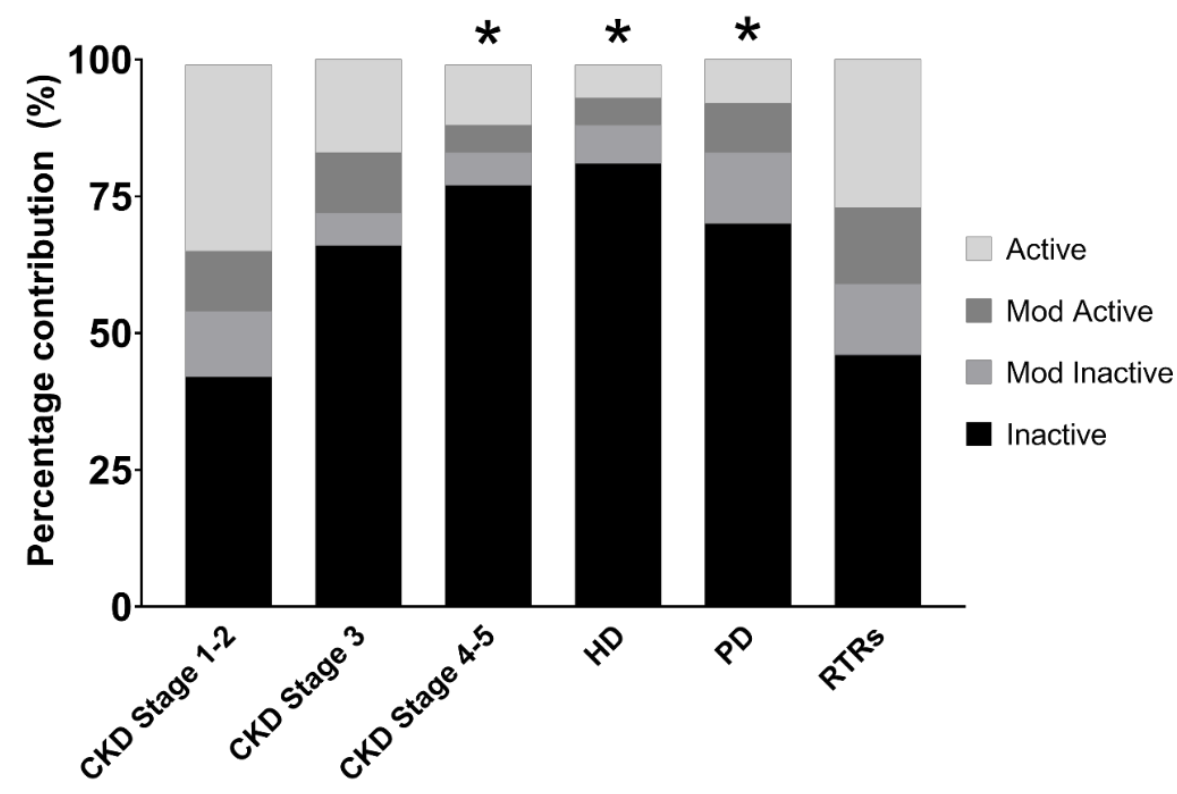

Physical activity status taken from General Practice Physical Activity Questionnaire (GPPAQ) adjusted to include $\geq 3$ hours of walking as 'active' (GPPAQ-WALK). CKD = chronic kidney disease; HD = haemodialysis; PD = peritoneal dialysis; RTRs = renal transplant recipients. Significant differences versus CKD Stage 1-2 (reference group) denoted by * and set at $P<0.001$; analysis adjusted for age and sex 
Figure 2. Frequency of physical activities reported

$\square$ CKD Stage 1-2 $\square$ CKD Stage $3 \square$ CKD Stage 4-5 $\square$ HD $\square$ PD $\square$ RTRs

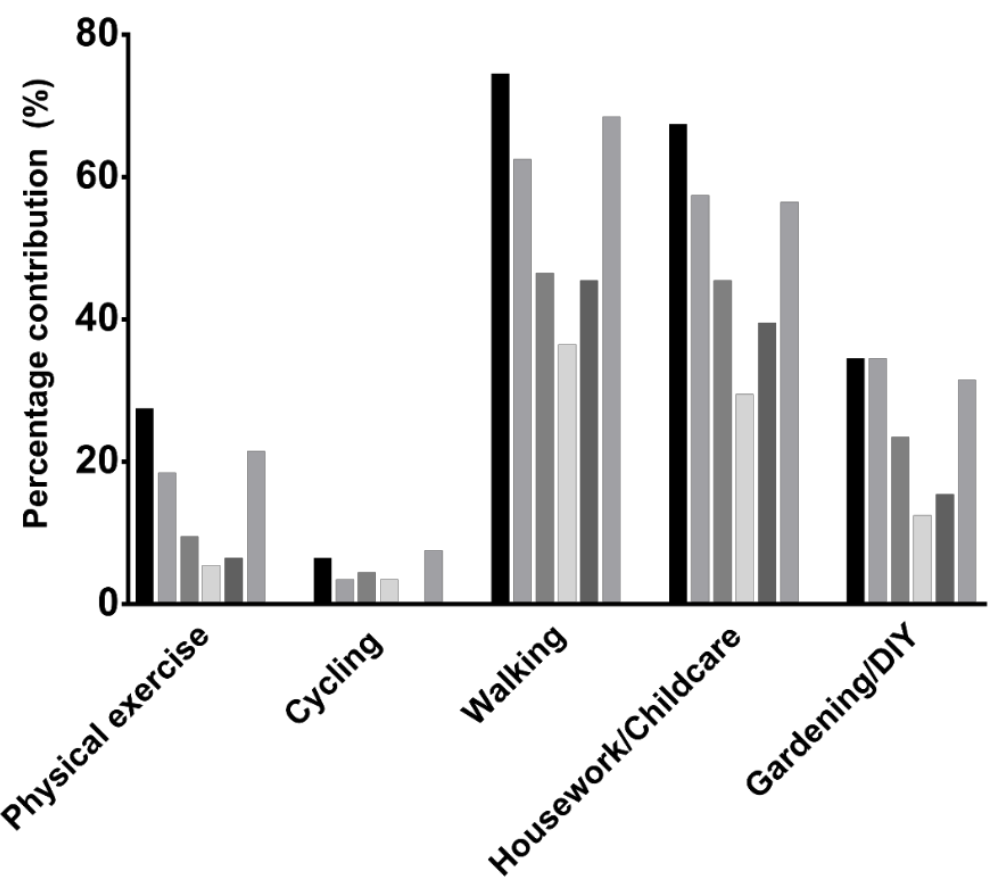

Physical activity status taken from General Practice Physical Activity Questionnaire (GPPAQ). CKD = chronic kidney disease; $\mathrm{HD}=$ haemodialysis; $\mathrm{PD}=$ peritoneal dialysis; RTRs = renal transplant recipients; DIY = 'Doit-yourself' e.g., maintenance or repair work 
Figure 3. Physical inactivity and significant disease group interactions

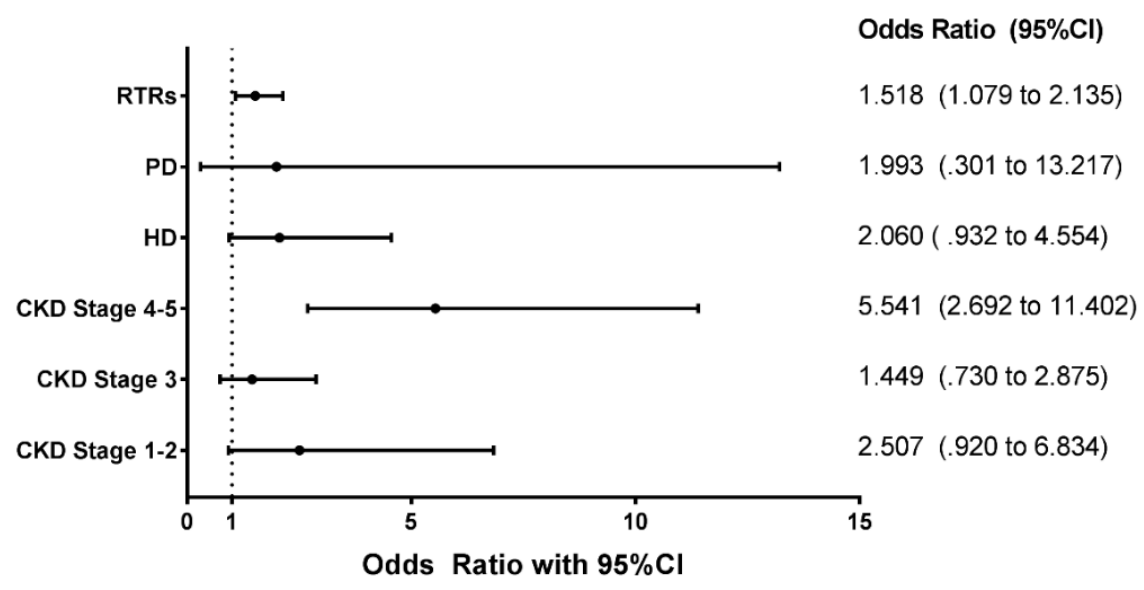

a) Age ( $\leq 61$ years (median) as reference)

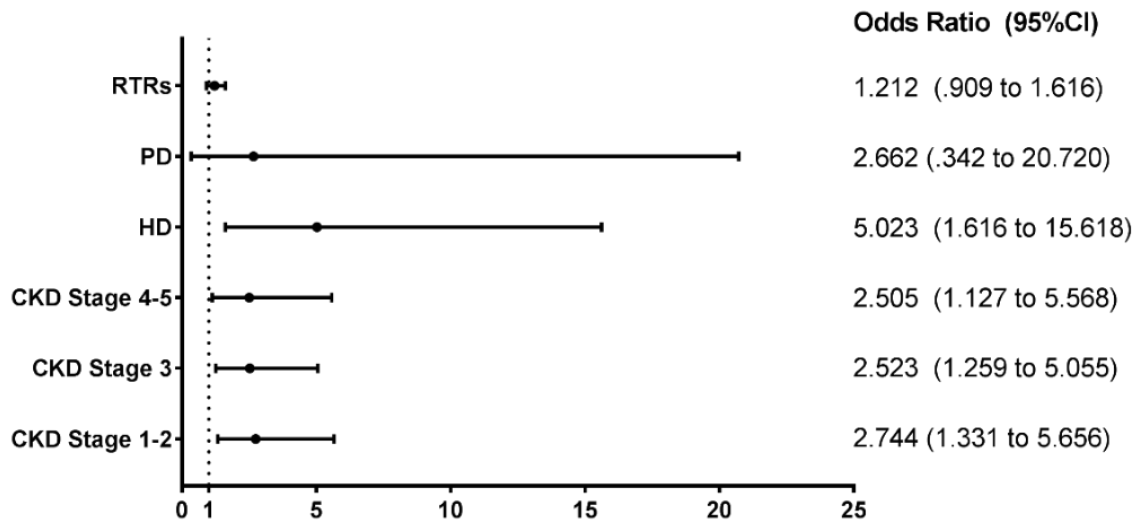

Odds Ratio with $95 \% \mathrm{Cl}$

b) Sex (male as reference)

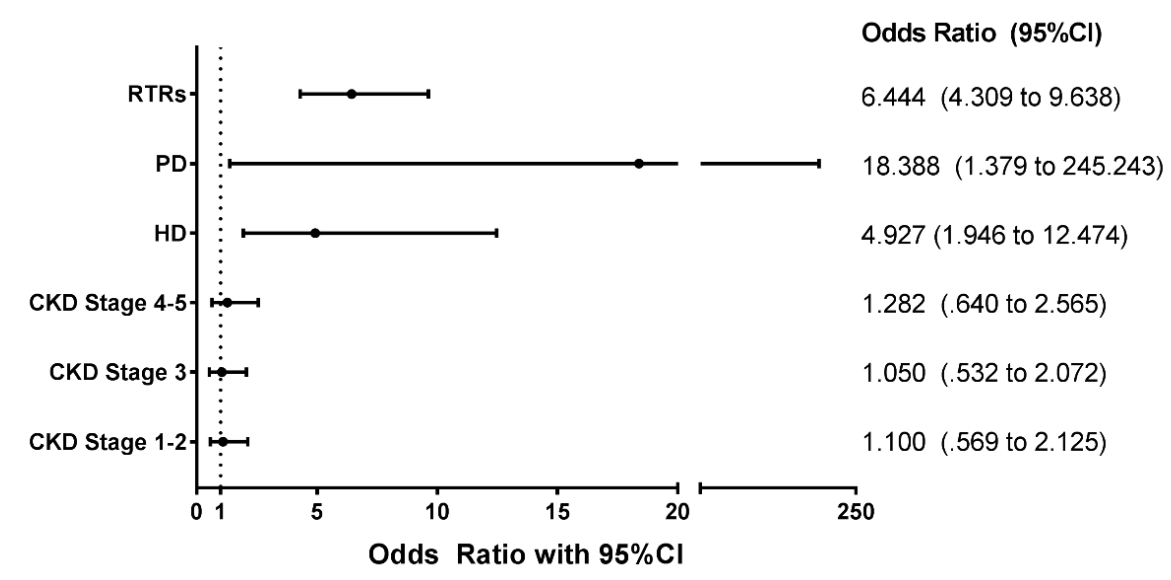

c) $\mathrm{VO}_{2}$ peak ( $>22.3 \mathrm{ml} \cdot \mathrm{kg} \cdot \mathrm{min}^{-1}$ (median) as reference)

Data displayed as mutually adjusted Odds Ratio (OR) with $95 \%$ confidence interval (CI); an OR of $>1$ indicates greater odds of being classified as physically 'inactive' compared to reference. Age and VO2peak coded as a dichotomous variables based on the median of the total sample population. $\mathrm{CKD}=$ chronic kidney disease; $\mathrm{HD}=$ haemodialysis; $\mathrm{PD}=$ peritoneal dialysis; $\mathrm{RTRs}=$ renal transplant recipients; $\mathrm{VO}_{2}$ peak = calculated from DASI 